\title{
Preparation and evaluation of biomimetric nano-hydroxyapatite-based composite scaffolds for bone-tissue engineering
}

\author{
YANG ChunRong $^{1 *}$, WANG YingJun ${ }^{2} \&$ CHEN XiaoFeng ${ }^{2}$ \\ ${ }^{1}$ Department of Materials Science and Engineering, Fujian University of Technology, Fuzhou 350108, China; \\ ${ }^{2}$ Key Laboratory of Specialty Functional Materials of the Ministry of Education, South China University of Technology, \\ Guangzhou 510640, China
}

Received September 21, 2011; accepted February 7, 2012; published online May 15, 2012

\begin{abstract}
In the present study, novel biomimetic composite scaffolds with a composition similar to that of natural bone were prepared, using nano-hydroxyapatite, collagen, and phosphatidylserine. The scaffolds possess an interconnected porous structure with a porosity of $84 \%$. The pore size ranges from several micrometers up to about $400 \mu \mathrm{m}$. In-vitro studies in simulated body fluids showed that the morphologies of the products derived from mineralization can be regulated by the extracellular matrix components of the scaffolds; this in turn leads to creation of a large number of hydroxyapatite crystals on the scaffold surface. The regulatory properties of collagen and phosphatidylserine also influenced the cell response to the composite scaffolds. MC3T3-E1 cells attached and spread on the surfaces of the materials and interacted with the substrates; this may be the result of charged groups on the composite materials. Radiological analysis suggested that calluses and bone bridges formed in defects within 12 weeks. These composite scaffolds may therefore be a suitable replacement in bone-tissue engineering.
\end{abstract}

composite, scaffold, cell, biomineralization, regulatory

Citation: Yang C R, Wang Y J, Chen X F. Preparation and evaluation of biomimetric nano-hydroxyapatite-based composite scaffolds for bone-tissue engineering. Chin Sci Bull, 2012, 57: 2787-2792, doi: 10.1007/s11434-012-5201-4

Tissue engineering is a promising approach in the area of bone repair and regeneration. The development of bone substitutes based on osteogenic growth and differentiation factors, with an optimal delivery system, has drawn considerable attention recently. Bone-like tissue-engineered composite materials with properties optimized for specific repair functions have been developed to meet these requirements, and promising results have been achieved [1-3]. However, such composites demonstrated no bone-like nanostructure and bone-tissue reactions. The structure of bone is very ingenious in maintaining strength and biological activity.

Hydroxyapatite (HA), which is the main inorganic component of bone, demonstrates exceptionally good biointegrative properties and osteoconductivity; osteoclastic cells appear around the material, and subsequently new bone is regenerated with the surrounding osteoblasts. However, some

*Corresponding author (email: lambmeo@163.com) clinical problems are observed with HA, e.g. it lacks osteoinductivity, and it is too brittle to be crushed, not only after implantation, but also in both tissue culture and surgical operations. Moreover, it takes more than a year to degrade completely, even after bone regeneration in the pores of the ceramic, and this increases the risk of fracture and inhibits further bone regeneration. Collagen (COL), which is the main organic component of bone, has the potential advantages of specific cell interactions and hydrophilicity. Osteogenesis generally starts with the production and release of COL fibrils from cells. A composite of HA and COL, the main components of bone, would be expected to have good bone-tissue reactions. Many researchers have therefore prepared HA/COL composites [4-8]. However, phosphatidylserine (PS) is an important component of cell velum lipid, and plays a key role in tissue development, repair, and function.

So far, no other studies of composites of HA, COL, and 
phosphoserine have been reported. The purpose of this study was to combine these three materials. The in-vitro and in-vivo responses of the biomimetic composites were assessed. The materials are intended to be used as scaffolds in bone-tissue engineering. The major advantages of this approach are its simplicity and low cost. This study also extends related recent research on composite materials based on biocompatible HA or bioresorbable bioglass-coated surgical implants $[1-4,12,13]$.

\section{Materials and methods}

\subsection{Materials}

Nano-HA (nHA) was synthesized using a sol-gel method. COL was purchased from Chunger Ltd. (Guangzhou, China). The COL was type I, prepared from bovine tendon in the form of an acetic acid solution with a content of $5.8 \mathrm{mg} / \mathrm{mL}$ and $\mathrm{pH}$ 5.0. PS was supplied by Boao Medical Ltd. (Shanghai, China).

Dulbecco's minimum essential medium (DMEM) and fetal bovine serum (FBS) were purchased from Sigma ( $\mathrm{St}$ Louis, MO, USA). Tissue-culture clusters and flasks were purchased from Corning (Midland, MI, USA). All other reagents were of analytical grade.

\subsection{Preparation of composite scaffolds}

The nHA-COL-PS composite scaffolds consisted of $65 \%$ parts by weight of inorganic components, i.e. nHA, and $35 \%$ parts by weight of organic components; the organic components consisted of $88.9 \mathrm{wt} \%$ COL and $11.1 \mathrm{wt} \%$ PS. The composite scaffolds were prepared using a freeze-drying technique. The nHA particles were gradually added to a solution of COL-PS and intensively mixed. Then 1-ethyl-3(3-dimethyl aminopropyl) carbodiimide (EDC) and N-hydroxysuccinimide (NHS) (4:1 ratio) were incorporated in deionized water at a content of $2.5 \mathrm{mg} / \mathrm{mL}$ and the $\mathrm{pH}$ was adjusted to 5.5 with 2-(4-Morpholino)ethanesulfonic acid (MES); the solution was kept at $4{ }^{\circ} \mathrm{C}$ for $24 \mathrm{~h}$ to allow cross-linking. The mixture was then transferred to a freezer (VXE380, Jouran, France) at $-60^{\circ} \mathrm{C}$ for $12 \mathrm{~h}$ to solidify the solvent and induce solid-liquid phase-separation. The solidified mixture was freeze-dried in a freeze-dryer (ALPHA2-4, Christ, Germany) at $-30^{\circ} \mathrm{C}$ for $24 \mathrm{~h}$ to form porous scaffolds.

\subsection{Biomineralization experiments}

Biomineralization experiments were performed to evaluate the biological properties of the composite scaffolds. The growth medium for biomineralization was simulated body fluid (SBF). It was prepared with the following ionic concentrations: $\left[\mathrm{Na}^{+}\right]=142.0 \mathrm{mmol} / \mathrm{dm}^{3},\left[\mathrm{~K}^{+}\right]=5.0 \mathrm{mmol} / \mathrm{dm}^{3},\left[\mathrm{Mg}^{2+}\right]=$ $1.5 \mathrm{mmol} / \mathrm{dm}^{3},\left[\mathrm{Ca}^{2+}\right]=2.5 \mathrm{mmol} / \mathrm{dm}^{3},\left[\mathrm{Cl}^{-}\right]=147.8 \mathrm{mmol} / \mathrm{dm}^{3}$, $\left[\mathrm{HCO}_{3}^{2-}\right]=42.0 \mathrm{mmol} / \mathrm{dm}^{3},\left[\mathrm{HPO}_{4}^{2-}\right]=1.0 \mathrm{mmol} / \mathrm{dm}^{3}$, and
$\left[\mathrm{SO}_{4}^{2-}\right]=0.5 \mathrm{mmol} / \mathrm{dm}^{3}$. The solution was buffered at $\mathrm{pH} 7.4$ with tris(hydroxymethyl)aminomethane $\left[\left(\mathrm{CH}_{2} \mathrm{OH}\right)_{3} \mathrm{CNH}_{2}\right]$ and $1 \mathrm{~mol} / \mathrm{L} \mathrm{HCl}$ at room temperature.

The samples were added to the system at $37^{\circ} \mathrm{C}$ and removed from the SBF at immersion times of 1,7 , and $15 \mathrm{~d}$, respectively. The reaction products were gently rinsed with distilled water and frozen at $-80^{\circ} \mathrm{C}$ for $12 \mathrm{~h}$. The frozen samples were then placed in Petri dishes and lyophilized at $-50^{\circ} \mathrm{C}$ for $24 \mathrm{~h}$ to dry them completely.

\subsection{Cell culture using nHA-COL-PS scaffolds as supports}

To study the osteoblast biocompatibility of the nHA-COLPS scaffolds in bone formation, MC3T3-E1 cells (Japan Riken Cell Collection) were used to test the materials.

Prior to the cell-culture assays, the scaffolds were sterilized by exposure to X-rays. As the experimental materials were not opaque, and would form a shadow under a microscope, they were crumbled into small pieces for easy observation.

The substrates containing the cells were suspended in a DMEM-10\%-FBS medium. The cell density was adjusted to $1.0 \times 10^{4}$ cells $/ \mathrm{mL}$, and $2 \mathrm{~mL}$ of cell suspension were seeded on the samples, placed on the bottom of a 24-well cell-culture plate. The cells were then left undisturbed in a humidified incubator $\left(37^{\circ} \mathrm{C}\right.$ and $\left.5 \% \mathrm{CO}_{2}\right)$ to allow them to attach to the samples. The medium was changed every $2 \mathrm{~d}$.

Random images of cells on each substrate were acquired using a phase-contrast microscope (Olympus IX 71, Tokyo, Japan).

\subsection{Surgical protocol and radiological examination}

The bone-tissue reactions of the composites were examined in animal tests using rabbit tibiae. Composites of area 15 $\mathrm{mm} \times 20 \mathrm{~mm}$ were used for the tests. Five rabbits $(2-2.5 \mathrm{~kg}$, 1-year-old) were sedated by intravenous administration of butorphanol tartrate $(0.2 \mathrm{mg} / \mathrm{kg})$ and midazolam $(0.02 \mathrm{mg} / \mathrm{kg})$. Anesthesia was achieved by administration of propofol ( $3 \mathrm{mg} / \mathrm{kg}$ ), and was maintained with $2 \%$ isoflurane in oxygen administered through an endotracheal tube. The composites were implanted into $25-\mathrm{mm}$ bone defects in the tibiae of each rabbit.

The bone growth was periodically observed using X-ray optical micrographs up to 12 weeks.

\subsection{Scanning electron microscopy observations and energy-dispersive $\mathrm{X}$-ray analysis}

Scanning electron microscopy (SEM; 30XLFEG, Philips, Eindhoven, The Netherlands) was used to observe the growth of calcium phosphate inside the COL matrix. The samples were sputter-coated with a layer of gold for observation at $10 \mathrm{kV}$.

Energy-dispersive X-ray (EDX; INCA, Oxford Instru- 
ments, Oxford, UK) analysis was carried out to evaluate the calcium phosphate chemical components and the $\mathrm{Ca}: \mathrm{P}$ ratio.

\subsection{X-ray diffraction analysis}

X-ray diffraction (XRD) (X'PertPRO, PANalytical, The Netherlands) was used to verify HA formation on the surface of the composite scaffolds treated in SBF, using a flat camera and $40-\mathrm{keV} \mathrm{Cu}-\mathrm{K} \alpha$ radiation. The samples were $2 \theta$ scanned from $10^{\circ}$ to $70^{\circ}$ with a scan speed of $15.24 \% \mathrm{~min}$.

\section{Results and discussion}

\subsection{Microstructures of nHA-COL-PS composite scaffolds}

The SEM micrograph in Figure 1 shows that the scaffolds possess an interconnected porous structure with porosity of $84 \%$. The pore size ranges from several micrometers up to about $400 \mu \mathrm{m}$. This structure could provide sufficient space for cell growth and extracellular matrix production.

\subsection{In-vitro studies in SBF}

The composite scaffolds were tested using in-vitro studies in SBF to determine their potential as tissue-engineering scaffolds. Figure 2 shows the biomineralization process after immersing nHA-COL-PS samples in SBF at $37^{\circ} \mathrm{C}$ for 1,7 , and $15 \mathrm{~d}$. At first, the newly formed mineral particles were seen to form a line. After $7 \mathrm{~d}$, regular mineral crystals occurred in the SBF; they were octahedral (Figure 2(b) and (c)). After immersion in SBF for $15 \mathrm{~d}$, these crystals developed into a continuous mineral layer, formed by coalescence of large crystals (Figure 2(d)). The precipitation of minerals is therefore continuous and dynamic. Macroscopic growth of the coalesced crystals is achieved by assembling a large number of growth units.

EDX analysis of the minerals revealed that the main elements of the mineral were carbon, oxygen, calcium, and phosphorus, as shown in Table 1 . The Ca:P molar ratio was 1.63 , close to the value of 1.67 for natural bone.

Elemental analysis showed that the minerals contain carbon. If the hydrogen content is ignored, the oxygen content can be calculated using the following equation:

$18.976 /(7.059+18.976+5.874+12.415) \times 100 \%=42.81 \%$.

The oxygen content of natural HA was calculated using

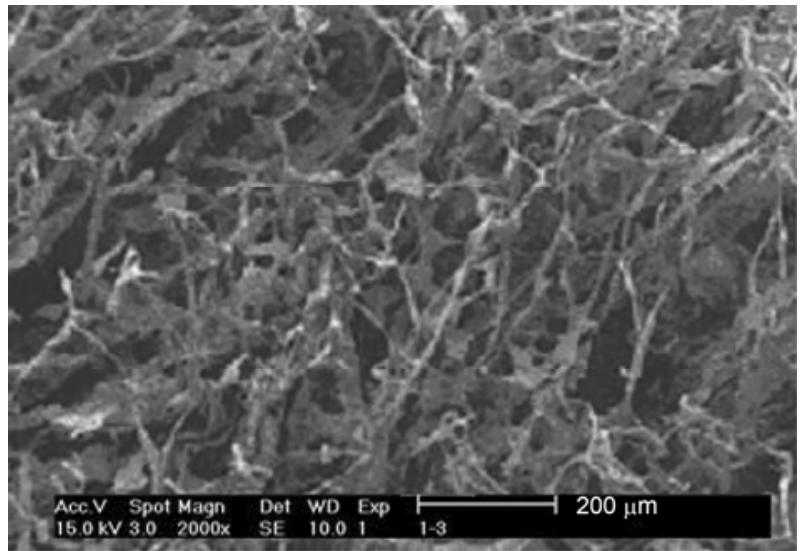

Figure 1 SEM micrograph of nHA-COL-PS scaffold.
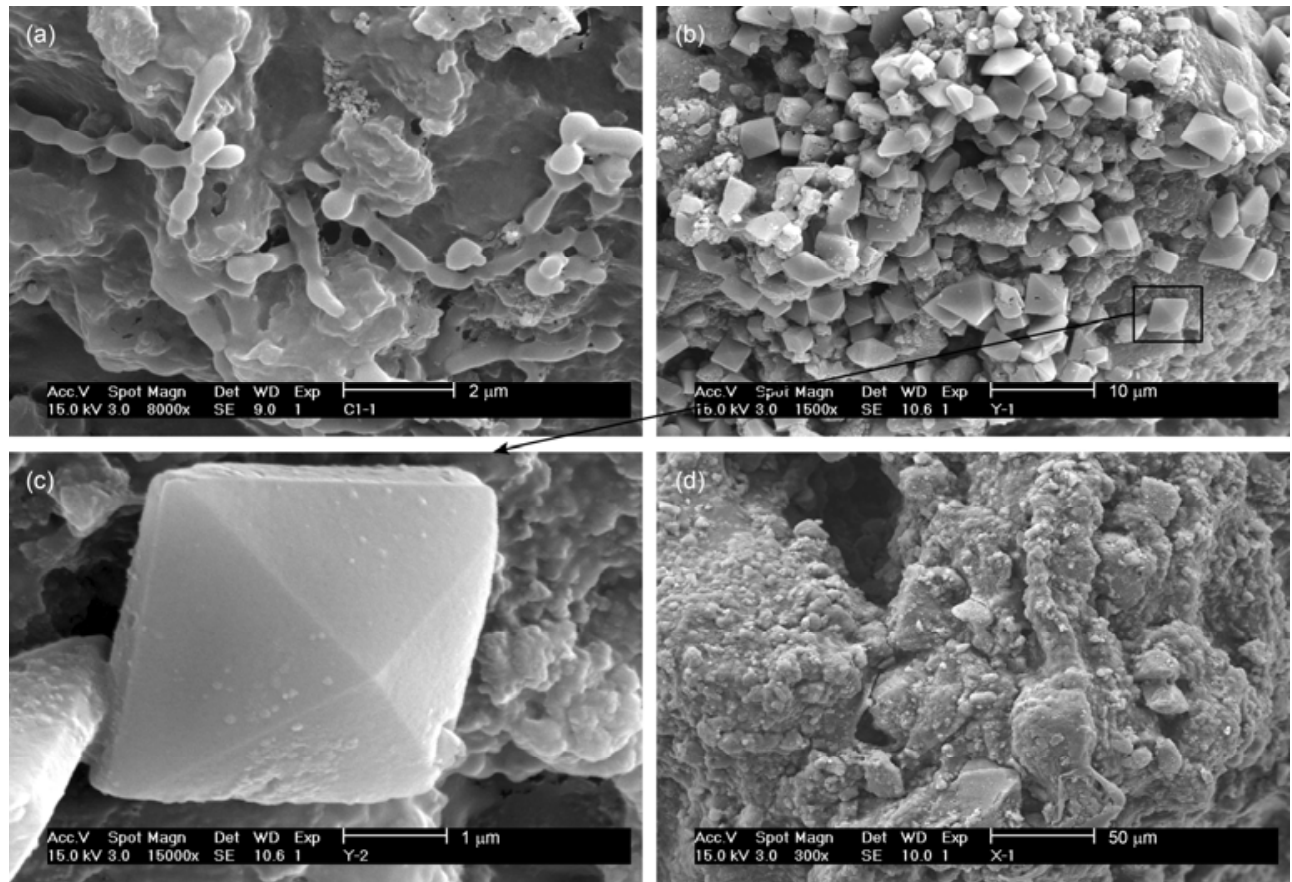

Figure 2 SEM micrographs of nHA-COL-PS scaffolds after biomineralization in SBF for $1 \mathrm{~d}(\mathrm{a}), 7 \mathrm{~d}$ (b), (c), and $15 \mathrm{~d}$ (d). 
Table 1 EDX analysis results for nHA-COL-PS scaffolds after biomineralization in SBF for $15 \mathrm{~d}$

\begin{tabular}{lcc}
\hline Element & Weight $(\%)$ & Atomic $(\%)$ \\
\hline $\mathrm{C}$ & 7.059 & 22.995 \\
$\mathrm{O}$ & 18.976 & 46.405 \\
$\mathrm{P}$ & 5.874 & 7.420 \\
$\mathrm{AuM}$ & 55.676 & 11.059 \\
$\mathrm{Ca}$ & 12.415 & 12.121 \\
Total & 100.000 & 100.000 \\
\hline
\end{tabular}

the same method. Taking the result of the calculation for natural HA of $41.52 \%$, which was lower than that of the mineralized product ( $42.81 \%)$, into account, the formed crystals were estimated to be carbonate-apatite.

The crystallinities of the structures formed on the mineralized composite samples were investigated using XRD; the results are shown in Figure 3. XRD confirmed the creation of a large number of HA crystals on the scaffold surface after immersion in SBF for $15 \mathrm{~d}$.

The mineralization process mentioned above can be considered as a self-assembly process, and it follows the principles of self-assembly processes. In biological systems, four stages are involved in biomineralization: supramolecular preorganization, interfacial molecular recognition, vectorial regulation, and cellular processing. The key point is that a surface with an organized arrangement of functional groups $\left(-\mathrm{OH},-\mathrm{PO}_{4} \mathrm{H}_{2},-\mathrm{NH}_{2}\right.$, etc.) resulting from organization of an organic matrix (COL, polysaccharides, etc.) acts as a template for the nucleation and growth of minerals from a supersaturated solution. The present study used COL and phosphoserine as self-assembly model systems to examine the possibility of synthesizing materials with such hierarchical structures, and nHA can provide sufficient inorganic ions as nucleation precursors.

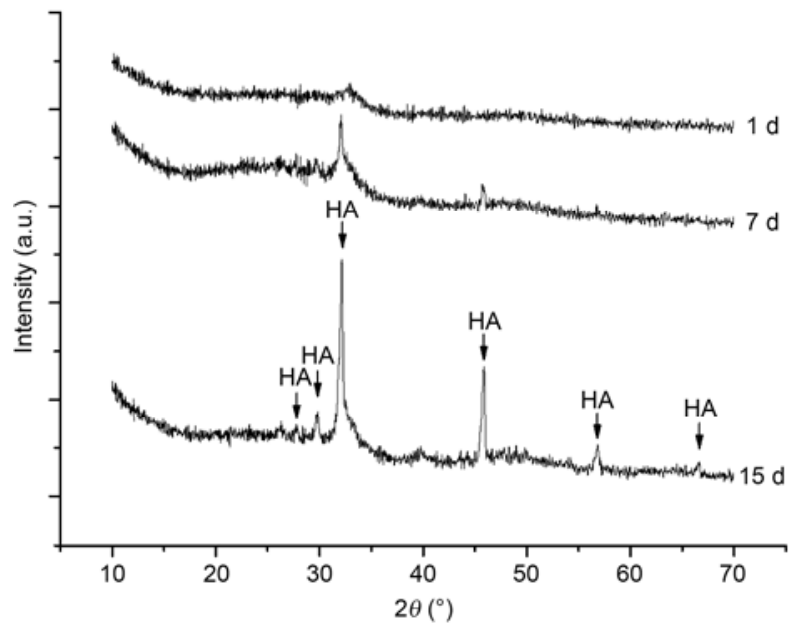

Figure 3 XRD patterns of nHA-COL-PS scaffolds after biomineralization in SBF for different times.
According to the basic mechanism of biomineralization, the control of nucleation and growth of calcium phosphate minerals by an organic matrix in aqueous media is complicated. In fact, most mineralization reactions occur not in solution but at interfaces. Organized functionalized surfaces provide high accessibility for nucleation reactions. The low energy-barrier to mobility in the plane of the surface can be used to facilitate the reactions, govern the reaction sites, orientate molecular recognition and site-specific nucleation, and regulate crystal growth.

Many groups have investigated the controlled nucleation and growth of crystals from organic templates in vitro, such as the study of in-vitro mineralization of bovine tendon COL by Iijima et al. [12]. Their study suggests that a regular arrangement of COL fibers has a close correlation with the orientation and growth of minerals. The electrochemical properties of $\mathrm{COL}$ can adjust the movement of inorganic ions within a matrix, thus affecting mineralization behavior. For example, with increasing $\mathrm{pH}$, the diffusion ability of $\mathrm{Ca}^{2+}$ ions within a matrix increases, and, conversely, the diffusion ability of $\mathrm{PO}_{4}^{3-}$ ions within the matrix decreases. Some other studies show a similar correlation between crystal growth and the crystallographic orientation of HA and the organic scaffold [13,14].

However, the COL matrix itself cannot completely fulfill the function of mineralization agent; it cannot initiate and guide the mineralization. These roles are performed by phosphorus proteins, which are rich in aspartic acid, by serine phosphorylation. Different biological systems select different matrices to perform biomineralization, but they all combine with $\mathrm{Ca}^{2+}$ via a phosphate functional group, followed by formation of calcium phosphate ceramics that have a mechanically strong bond to the matrix surface. PS plays an important part in the regulation of the mineralization process. It can interact with $\mathrm{Ca}^{2+}$ and $\mathrm{PO}_{4}^{3-}$ to form $\mathrm{Ca}-$ $\mathrm{PS}-\mathrm{PO}_{4}$ complexes, inducing $\mathrm{HA}$ to precipitate under substable conditions. Once the complex has been formed, almost no factor can hinder further formation of stable HA crystals. There are also electrostatic forces between the ions and groups in the scaffold material, such as $\mathrm{COO}^{-}$and $\mathrm{NH}_{4}^{+}$ groups of PS and COL, which result in local supersaturation followed by crystal nucleation.

\subsection{Cell biocompatibility of nHA-COL-PS scaffolds}

MC3T3-E1 cells were seeded on nHA-COL-PS substrates and then analyzed for their interactions with the substrates. Representative morphologies obtained are shown in Figure 4. When observed $12 \mathrm{~h}$ after seeding, the cells appeared with a narrow cell body and extended overlapping processes (Figure 4(a)). After $24 \mathrm{~h}$ culturing, the observations suggested that the cells growing on the substrates have proper cell-cell contacts with neighboring cells, and the cell processes are bridging across substrates or extending by forming a mesh in the substrates (Figure 4(b)). From the images, it is concluded 

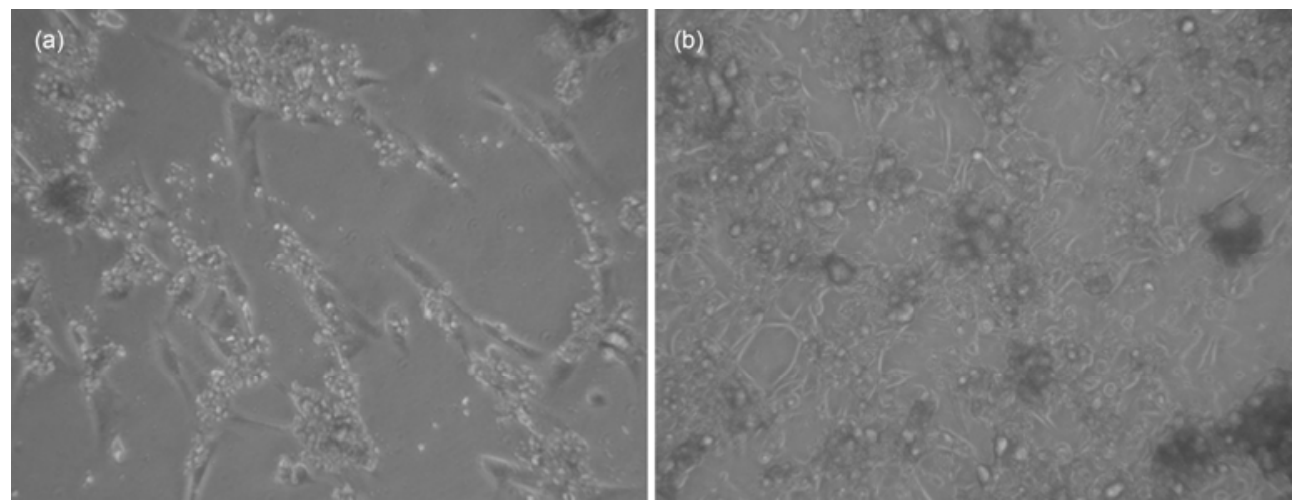

Figure 4 Optical micrographs of cells after $12 \mathrm{~h}$ (a) and $24 \mathrm{~h}$ (b) of culturing with nHA-COL-PS substrates.
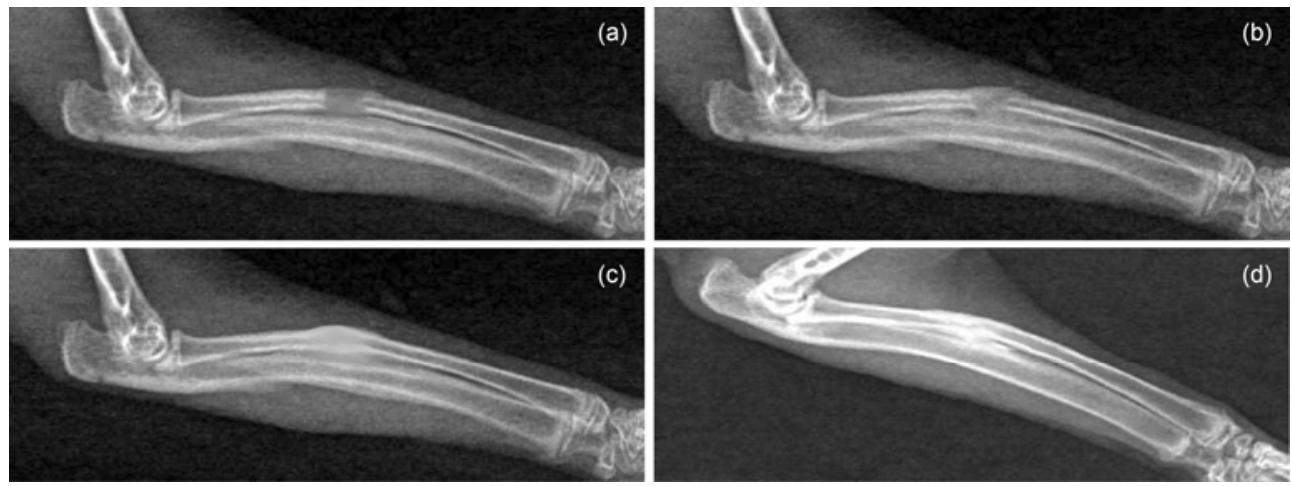

(d)

Figure 5 X-ray optical micrographs of bone defects filled with nHA-COL-PS scaffolds 2 weeks (a), 4 weeks (b), 8 weeks (c), and 12 weeks (d) after implantation.

that after culturing for $24 \mathrm{~h}$, the nHA-COL-PS substrates had good cell biocompatibility.

Tissue engineering aims to design functionally active cells within supporting bioscaffolds to promote the development of new tissues. Adhesive interactions between cells and nHA-COL-PS materials provide signals that influence the cellular state. Analysis of cell interactions with the substrates is necessary to regulate both cell expansion in the cell-loaded tissue-engineered constructions and their subsequent in-vivo integration with the surrounding tissues.

The initial adhesion and growth of osteoblasts on implant surfaces requires the contribution of charged functional groups, acting as a primary mechanism in regulating cellmatrix interactions. The substrate composition probably plays an important role in the actions of the analyzed cells. COL is a cell-adhesion molecule with large numbers of amino and carboxyl groups on the side chains. It is involved in amplification of integrin-mediated cell adhesion, maintenance of adherent junction integrity, and control of extension events, facilitating the interaction of cells with neighboring cells and the matrix. PS is an important component of cell velum lipid, and plays a key role in cell differentiation, proliferation, and regeneration. In an alkaline environment, there are negatively charged phosphate groups $\left(-\mathrm{PO}_{4} \mathrm{H}_{2}\right)$ in the PS molecules. These phosphate groups of PS can critically affect the biological activities of a variety of membrane-bound proteins and receptors.

\subsection{Radiological evaluation}

In the present study, bone-bridge formation was examined. An interface between the implant and the recipient bone was not clearly visible after 2 weeks, and callus formation was poorly visible (Figure 5(a)). After this period, a faint bone-bridging-like shadow could be observed (Figure 5(b)). In addition, thick new bone coverage adjacent to the implant, i.e. anterior bone bridging, could be observed after 8 weeks (Figure 5(c)). Absorption of the newly formed bone did not occur, and instead bone maturation continued until 12 weeks after the operation (Figure 5(d)).

In contrast, it was found that bone defects without a filler did not show any evidence of bone formation at 12 weeks after the operation (Figure 6).

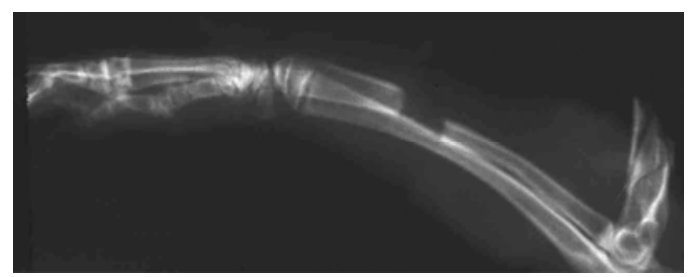

Figure 6 X-ray optical micrograph of bone defects without filler 12 weeks after implantation. 
We concluded that, in the present study, nHA-COL-PS scaffolds produced excellent results with respect to bone formation. However, further studies of the composites for longer time periods and at different anatomical sites are needed to identify bioresorption, which can act as a cell carrier for clinical applications.

This work was supported by Science and Technology Plan Project of Fujian Department of Education, China (JK2009021).

1 Place E S, Evans N D, Stevens M M. Complexity in biomaterials for tissue engineering. Nat Mater, 2009, 8: 457-470

2 Li Z H, Huang Y F, Ma X, et al. Biocompatibility of hydroxyapatite surface modified titanium skirt for keratoprosthesis: Scanning electron microscopy observation. J Clin Tissue Eng Res, 2010, 14: 5346-5350

3 Zhao J, Lu X, Duan K, et al. Improving mechanical and biological properties of macroporous HA scaffolds through composite coatings. Colloids Surf B Biointerfaces, 2009, 74: 159-166

4 Feng W P, Qi Y M, Tang K Y. Preparation and characterization of collagen-hydroxyapatite/gum Arabic composite (in Chinese). Acta Mater Comp Sin, 2010, 27: 113-119

5 Tcacencu I, Wendel M. Collagen-hydroxyapatite composite enhances regeneration of calvaria bone defects in young rats but postpones the regeneration of calvaria bone in aged rats. J Mater Sci, 2008, 19: 2015-2021

6 Wang X, Wang X, Tan Y, et al. Synthesis and evaluation of collagenchitosan-hydroxyapatite nanocomposites for bone grafting. J Biomed Mater Res, 2009, 89: 1079-1087

7 Lan X Y, Zhou C S, Tian J. Repair of radius bone defect with the nano-hydroxyapatite/collagen/calcium alginate (in Chinese). Acta Med Sin, 2009, 31: 459-463

8 Teng S H, Lee E J, Wang P, et al. Three-layered membranes of collagen/ hydroxyapatite and chitosan for guided bone regeneration. J Biomed Mater Res, 2008, 87: 132-138

9 Michael R, Charoenrook V, de la Paz M F, et al. Long-term functional and anatomical results of osteo- and osteoodonto-keratoprosthesis. Graefes Arch Clin Exp Ophthalmol, 2008, 246: 1133-1137

10 Bonnier F, Rubin S, Debelle L, et al. FTIR protein secondary structure analysis of human ascending aortic tissues. J Biophot, 2008, 1: 204-214

11 Viitala R, Franklin V, Green D, et al. Towards a synthetic osteo-odonto-keratoprosthesis. Acta Biomater, 2009, 5: 438-452

12 Iijima M, Moriwaki Y, Kuboki Y. Oriented and lengthwise growth of octacalcium phosphate on collagenous matrix in vitro. Conn Tissue Res, 1997, 36: 51-61

13 Chang S H, Hsu Y M, Wang Y J, et al. Fabrication of pre-determined shape of bone segment with collagen-hydroxyapatite scaffold and autogenous platelet-rich plasma. J Mater Sci, 2009, 20: 23-31

14 Ciolino J B, Dohlman C H. Biologic keratoprosthesis materials. Int Ophthalmol Clin, 2009, 49: 1-9

Open Access This article is distributed under the terms of the Creative Commons Attribution License which permits any use, distribution, and reproduction in any medium, provided the original author(s) and source are credited. 